\title{
MISSIONS CHINOISES
}

Gianni CRIVELleR, Preaching Christ in late Ming China. The Jesuits' presentation of Christ from Matteo Ricci to Giulio Aleni. Taibei/Brescia, Ricci Institute, 1997. $15 \times 23,479$ p., bibliogr. (Variétés sinologiques, 86; Fondazione Civiltà Bresciana Annali, 10).

Martino Martini. A humanist and scientist in seventeenth-century China. Éd. Franco Demarchi et Riccardo SCARTEzzinI. Trente, Università degli studi di Trento, 1996. $16 \times 24$, xIV-381 p.

Martino Martini. Opera Omnia. Éd. Franco DemarchI. Vol. I : Lettere e documenti; vol. II : Opere minori. Éd. Giuliano BerTuccioli. Trente, Università degli studi di Trento, 1998-. $17 \times 24,545$ p. et $516 \mathrm{p}$.

Peter ENGELfRIEt, Euclid in China. The genesis of the first Chinese translation of Euclid's Elements Books I-VI (Jihe yuanben; Beijing, 1607) and its reception up to 1723. Leyde, E. J. Brill, 1998. 16,5 × 24, 488 p., bibliogr., index.

Isaia IANNACCONE, Johann Schreck Terrentius. Le science rinascimentali e lo spirito dell'Accademia dei Lincei nella Cina dei Ming. Naples, Istituto universitario orientale, Dipartimento di studi asiatici, 1998. 17,5 × 25, 147 p., bibliogr. (Series Minor, LIV).

Jinshui LiN, Li Madou yu Zhongguo (M. Ricci et la Chine). Beijing, Zhongguo shehui kexue chubanshe, 1996. $14 \times 20$, Ix-337 p.

"Scholar from the West. » Giulio Aleni, S.J. (1582-1649) and the dialogue between christianity and China. Éd. Tiziana LiPPIELLo et Roman MALEK. Nettetal, Steyler Verlag, 1997. 16,5 × 24, Xxvi-671 p., bibliogr., index (Monumenta serica monograph series, XLII; Fondazione Civilità Bresciana Annali, IX).

Western learning and christianity in China. The contribution and impact of Johann Adam Schall von Bell, S.J. (1592-1666). Éd. Roman MALEK, 2 vol. Nettetal, Steyler Verlag, 1999. 16,5 × 24, xLII-1259 p., bibliogr., index (Monumenta serica monograph series, XXXV).

Western humanistic culture presented to China by Jesuit missionaries (xvith-xVIIth centuries). Proceedings of the conference held in Rome, Oct. 25-27, 1993. Éd. Federico MASINI. Rome, Institutum Historicum Societatis Iesu, 1996. $16 \times 24$, 396 p., index (Bibliotheca Instituti Historici S.I., XLIX).

Alvarez Semedo, Histoire universelle du Grand Royaume de la Chine. Trad. JeanPierre Duteil. Paris, Kimé, 1996. 14,5 × 21, 233 p.

Revue de synthèse : $4^{\mathrm{e}}$ S. $\mathrm{n}^{\text {os }}$ 2-3, avr.-sept. 1999, p. 486-491. 
Xujiahui zangshulou MingQing tianzhujiao wenxian (Chinese christian texts from the Zikawei Library). Éd. Nicolas Standaert, Ad Dudink, Yi-Long Huang et Ping-Yi CHu, 5 vol. Taibei, Fu Jen Catholic University Press, 1996. $15 \times 21,5$, $2621 \mathrm{p}$.

Kai Zhang, Pang Diwo yu Zhongguo (Diego de Pantoja et la Chine). Beijing, Beijing tushuguan chubanshe, $1997.14 \times 20,466 \mathrm{p}$.

L'historiographie récente sur les jésuites en Chine se caractérise par deux tendances majeures. D'une part, elle se situe dans la lignée des méthodes et des centres d'intérêts caractéristiques du $\mathrm{xx}^{\mathrm{e}}$ siècle. D'autre part, elle subit l'influence d'une recherche historique centrée sur la réception par la culture locale.

La première approche, dite « classique », favorise l'étude du missionnaire. Durant les années 1980 et 1990, ont été organisés plusieurs colloques d'anniversaire dédiés à un missionnaire jésuite : Ferdinand Verbiest (1623-1688), Leuven, 1988; Johann Adam Schall von Bell (1592-1666), Sankt Augustin, 1992; Giulio Aleni (15821649), Brescia, 1994; Martino Martini (1664-1661), Pékin, 1994. Chacun a donné lieu à une publication ${ }^{1}$. En général, ces volumes abordent un grand nombre d'aspects de la vie du personnage concerné et proposent de le concevoir autrement que comme simple jésuite, mais aussi comme diplomate, humaniste, ingénieur, astronome, etc. Le chercheur non averti aura cependant quelques difficultés à s'orienter dans ces volumes. Ils contiennent des articles s'appuyant sur des méthodes solides, suivant la meilleure tradition de cette approche classique, ainsi que des articles relevant de la seconde tendance, mais malheureusement ils y trouveront aussi des articles qui répètent les informations des sources secondaires bien connues.

La poursuite de cette approche classique permet aussi quelques nouveautés. À côté des personnages les plus renommés, des études sont ouvertes sur certains jésuites moins connus comme l'astronome Diego de Pantoja (1571-1618) dont le traité sur « les sept péchés et sept vertus » a connu une certaine influence ${ }^{2}$, le botaniste et médecin Johann Schreck Terrentius (1576-1630) ${ }^{3}$ et Jean-François Gerbillon (1654-1707), un des cinq mathématiciens envoyés en Chine par Louis XIV ${ }^{4}$. On peut aussi se référer aux articles sur des jésuites publiés dans les actes du $\mathrm{VI}^{\mathrm{e}}$ et du VII $^{e}$ colloque international de Sinologie de Chantilly ${ }^{5}$ ainsi qu'au numéro spécial de

1. Ferdinand Verbiest (1623-1688), Jesuit missionary, scientist, engineer and diplomat, éd. John W. Wrrek, Nettetal, Steyler Verlag, 1994; Western learning and christianity in China, op. cit. supra, p. 486; « Scholar from the West », op. cit. supra, p. 486; Eugenio MENEGoN, Un solo Cielo; Giulio Aleni (1582-1649). Geografia, arte, scienza, religione dall'Europa alla Cina, Brescia, Grafo, 1994; Martino Martini. A humanist and scientist in seventeenthcentury China, op. cit. supra, p. 486.

2. Voir Kai ZHANG, op. cit. supra, p, 487.

3. Voir I. IANNACCONE, op. cit. supra, p. 486; voir aussi Georges MÉTAllÉ, «Des manuscrits en quête d'auteur. Du Plinius Indicus de Johan Schreck au Bencao gangmu de Li Shizhen et au Bencao pinhui jingyao de Liu Wentai ", Journal asiatique, t. CCLXXXVI, 1, 1998, p. 259-281.

4. $\mathrm{M}^{\mathrm{me}}$ Yves de Thomaz de Bossierre, Jean-François Gerbillon, S.J. (1654-1707). Un des cinq mathématiciens envoyés en Chine par Louis XIV, Leuven, Ferdinand Verbiest Foundation, 1994.

5. Actes du $\mathrm{VI}^{\mathrm{e}}$ colloque international de Sinologie de Chantilly, 11-14 sept. 1989, Images de la Chine. Le contexte occidental de la sinologie naissante, éd. Edward MALATESTA et Yves Raguin, Taibei/Paris, Institut Ricci, 1995. Actes du VII ${ }^{\mathrm{e}}$ colloque international de Sinologie de 
la revue de l'institut culturel de Macao ${ }^{6}$. Importantes sont également les éditions et/ou traductions (bien annotées) de sources telles que l'Astronomia Europaea de Verbiest ${ }^{7}$, les lettres de Karl Slavícek (1678-1735) et le grand projet concernant les cuvres complètes de Martini ${ }^{8}$. Enfin, il faut noter, dans le domaine de l'archéologie religieuse, les publications, bien illustrées, sur les tombeaux des jésuites à Beijing ${ }^{9}$.

Sous la même rubrique classique, trois nouveaux livres tentent d'écrire une histoire générale des jésuites en Chine. Andrew Ross ${ }^{10}$ essaie de montrer comment la vision d'Alessandro Valignano et Matteo Ricci a été " trahie " ( betrayed") dans la suite de l'histoire des jésuites en Chine tandis que Jean-Pierre Duteil ${ }^{11}$, à la suite du travail de Jean Delumeau qui préface ce livre, soumet l'histoire à de nouvelles questions (statistiques, géographie, etc.). Malheureusement, les deux livres occultent le point de vue chinois, voire même dans le second cas, souffrent d'imprécisions et révèlent des lacunes bibliographiques, notamment anglophones ${ }^{12}$. Des références à des textes chinois sont aussi absentes dans le chef d'œuvre de Dauril Alden ${ }^{13}$, mais par sa lecture transversale de la province jésuite portugaise dans toute son extension, Chine incluse, l'auteur offre une vue d'ensemble tout à fait neuve, basée sur une recherche conduite avec beaucoup de rigueur. Le livre contient une longue partie sur les finances des missions, un aspect qu'il va falloir examiner davantage dans l'avenir.

En Chine populaire, on a également vu apparaître un grand nombre d'articles sur les jésuites qui se situent dans la même approche ${ }^{14}$. Même si la plupart de ces articles apportent relativement peu d'éléments nouveaux, et se limitent aux sciences,

Chantilly, 8-10 sept. 1992, Échanges culturels et religieux entre la Chine et l'Occident, éd. Edward Malatesta, Yves Raguin et Ad DudinK, Taibei/Paris, Institut Ricci, 1995.

6. "The Jesuits, 1594-1994. Macao and China, East Meets West », numéro spécial de la revue Review of culture, 21, 1994.

7. Noël Golvers, The Astronomia Europaea of Ferdinand Verbiest, S.J., (Dillingen, 1687). The text, translation, notes and commentaries, Nettetal, Steyler Verlag (Monumenta serica monograph series, XXVIII), 1993.

8. Voir Karel Slavicek: Listy z Ciny do vlasti a jina korespondence s evropskymi hvezdari (1716-1735) Letters from China to his native country and other correspondence with European astronomers (1716-1735), Trad. et éd. Josef Kolmas Prague, Vysehrad Publ., 1994; voir aussi Martino Martini. Opera Omnia, op. cit. supra, p. 486.

9. Lishi yihen. Li Madou ji Ming Qing xifang chuanjiaoshi mudi (Les tombeaux de M. Ricci et des missionnaires occidentaux des Ming et des Qing), éd. Zhiyu GAo et al., Beijing, Zhongguo Renmindaxue chubanshe, 1994; Departed, Yet Present. Zhalan, The oldest Christian cemetry in Beijing, éd. E. Malatesta et Zhiyu GAO, Macau, Instituto cultural, 1995.

10. Andrew Ross, $A$ vision betrayed. The Jesuits in Japan and China, 1542-1742, Édimbourg, Edinburgh University Press, 1994.

11. Jean-Pierre DuTEIL, Le Mandat du Ciel. Le rôle des jésuites en Chine, de la mort de François-Xavier à la dissolution de la Compagnie de Jésus (1552-1774), Paris, Arguments, 1994.

12. Voir aussi J. W. Wrrek, "Conspectus Bibiographici. The Jesuits in China during the seventeenth and eighteenth centuries », Archivum Historicum Societatis Iesu, t. LXV, 2, 1996, p. 233-244.

13. Dauril Alden, The Making of an enterprise. The Society of Jesus in Portugal, its empire, and beyond, 1540-1750. Stanford, CA, Stanford University Press, 1996. Pour une analyse de cet ouvrage à partir du point de vue américain, voir le compte rendu d'Aliocha MaLDAVSKY, supra, p. 478-481.

14. Voir aussi Paola Calanca, "La Chine populaire face aux jésuites (1582-1723). Le début d'une réévaluation historique ", in Images de la Chine, op. cit. supra n. 5, p. 193-212. 
leur nombre indique bien un renouveau de l'intérêt pour les questions d'échange entre l'ouest et l'est, rubrique sous laquelle ils apparaissent généralement. On peut noter cependant certains domaines originaux de recherche, comme par exemple la géographie $^{15}$, et souligner également la réédition et la traduction en chinois de deux grands œuvres de référence sur les biographies des jésuites, celles de Louis Pfister et de Joseph Dehergne ${ }^{16}$.

Parallèlement à cette approche classique, se dégagent plusieurs nouvelles approches influencées par une historiographie émanant d'autres domaines ${ }^{17}$. On peut regrouper ici ces approches sous trois rubriques.

En premier lieu, il devient important d'étudier davantage le contexte chinois dans lequel s'est déployée l'expérience jésuite. Jinshui $\operatorname{Lin}^{18}$ ou Ad Dudink ${ }^{19}$, par exemple, ont situé Ricci ou Aleni dans le réseau des relations chinoises. Dans la même optique se situent les recherches conduites sur les textes en chinois (et non plus seulement en langues occidentales) écrits par ou sur les jésuites. On peut noter un intérêt pour les textes « humanistes », traitant de sujets comme la dialectique ou l'éthique d'Aristote, ou le syllogisme et la méthode de comparaison ${ }^{20}$. Sur ces différents points, on peut notamment se référer aux études menées par un groupe de recherche européen dont deux volumes de travaux ont déjà été publiés ${ }^{21}$. Autre élément de ce renouveau historiographique, la parution d'articles ou de livres en langue occidentale sur des jésuites chinois, comme par exemple Wu Li (1632-1718),

15. Voir Zhongguo gudai dituji : Mingdai, éd. Wanru CaO, Beijing, Wenrou, 1995.

16. Voir Louis Pfister (Fei LAIZH), Zaihua yesuhuishi liezhuan ji shumu, trad. Chengjun Feng, 2 vol., Beijing, Zhonghua shuju, 1995 = nouv. éd. de Ruhua yesuhuishi liezhuan, Taibei, Commercial Press, $1960=$ trad. de Notices biographiques et bibliographiques sur les jésuites de l'ancienne mission de Chine (1552-1773), Shanghai, Mission catholique, 1932. Voir, également, Joseph Dehergne (Zhenhua Rong), Zaihua yesuhuishi liezhuan ji shumu bubian, trad. Sheng GeNG, 2 vol., Beijing, Zhonghua shuju, 1992 = trad. de Répertoire des jésuites de Chine de 1552 à 1800, Rome/Paris, Institutum Historicum Societatis Iesu, 1973.

17. Pour ce changement, voir Paul RuLE, «Chinese-Centered mission history ", in Historiography of the Chinese Catholic Church, éd. Jerome HeYndRICkx, Leuven, Ferdinand Verbiest Foundation, 1994, p. 52-59; Erik ZURCHER, \& From Jesuit studies to Western learning ", in Europe studies China. Papers from an international conference on the history of European sinology, Londres, Han Shan Tang Books, 1995, p 264-279; Daniel BAYs, "Preface ", in Christianity in China. From the eighteenth century to the present, éd. D. BAYs, Stanford, Stanford University Press, 1996; Nicolas StandaERT, « New trends in the historiography of Christianity in China », Catholic Historical Review, t. LXXXIII, 4, 1997, p. 573-613.

18. Voir, particulièrement, p. 286-316, in op. cit. supra, p. 486.

19. Voir "Giulio Aleni and Li Jiubiao ", p. 129-220, in «Scholar from the West », op. cit. supra, p. 486.

20. À titre d'exemple, E. ZürchER, "Renaissance rhetoric in late Ming China. Alfonso Vagnoni's Introduction to his Science of comparison», p. 331-360, in Western humanistic culture, op. cit. supra, p. 486; voir égaiement la réédition de sources premières par N. STANDAERT et al., op. cit. supra, p. 487.

21. L'Europe en Chine. Interactions scientifiques, religieuses et culturelles aux xvif et $x_{\text {XvI }}^{e}$ siècles, éd. Catherine Jami et Hubert Delahaye, Paris, Collège de France (Mémoires de l'Institut des hautes études chinoises, XXXIV), 1993; Western humanistic culture, op. cit. supra, p. 486; pour un compte rendu du troisième colloque intitulé « Between Ming and Qing. The Jesuits, dynastic decline, internal strife, and the establishment of a new order in seventeenth-century China », voir Sino-Western Cultural Relations Journal, t. XX, 1998, p. 73-76. 
peintre et poète au début des Qing ${ }^{22}$. Mais l'historiographie n'a pas encore totalement pris en compte leur rôle, alors que, au milieu du xvir' siècle, un tiers des jésuites en Chine étaient Chinois. Ce déplacement des études jésuites en direction du contexte chinois soulève une nouvelle question fondamentale: les jésuites étaient-ils, comme on l'a pensé jusqu'à présent, les protagonistes de leur propre entreprise ou faut-il considérer que ce qu'ils sont devenus en Chine a été largement influencé par ce contexte chinois ${ }^{23}$ ?

Un second changement se situe au niveau de la méthodologie et des questions concernant l'histoire de la transmission des idées et plus particulièrement les sciences. À partir de la question « Pourquoi les sciences modernes ont-elles éclos en Europe et non pas en Chine? ", la célèbre collection de Joseph Needham, «Science and civilisation in China ", (Cambridge, Cambridge University Press, 1954-) a eu une influence considérable sur la recherche qui visait à évaluer «l'influence » et «l'effet » des sciences apportées par les jésuites. Mais, comme le montre clairement le travail approfondi de Peter Engelfriet, les chercheurs actuels développent moins cet aspect «d'effet » et s'intéressent davantage aux différentes modalités de réception et d'appropriation des idées. Également importante du point de vue de la méthode est l'approche de Catherine Jami ${ }^{24}$. Celle-ci combine deux éléments, l'arrière-plan mathématique des jésuites en Europe et le public chinois auquel ces œuvres étaient destinées. En analysant trois versions de la géométrie euclidienne (1607, 1631, 1723), C. Jami montre l'évolution de la transmission de la géométrie. Une telle approche souligne que l'étude de la reproduction du savoir demande l'interaction de différentes disciplines. Un autre auteur productif dans ce domaine de la réception des sciences en Chine est Yi-long Huang, qui publie surtout en chinois citant un grand nombre de sources premières ${ }^{25}$.

Un troisième axe de la recherche commence à se dessiner progressivement. Jusqu'à présent, les études sur les jésuites en Chine étaient centrées sur les jésuites de la cour et leurs correspondants appartenant à l'élite. Mais cette élite ne consistait qu'en une petite minorité de chrétiens en Chine, et la majorité des jésuites était engagée dans un travail de pastorale et de catéchèse. Dans ces milieux, ils appliquaient des méthodes tout à fait différentes de celles mises en œuvre dans la transmission des sciences ou de la philosophie, domaines dont les élites chinoises se désintéressèrent d'ailleurs au cours du xvII ${ }^{e} \operatorname{siècle}^{26}$. L'intérêt des chercheurs se

22. Jonathan Chaves, Singing of the source. Nature and God in the poetry of the Chinese painter $W u L i$, Honolulu, University of Hawaii Press, 1993.

23. Voir Erik ZÖCHER, « Jesuit accommodation and the Chinese cultural imperative », in The Chinese rites controversy. Its history and meaning, éd. David E. Mungeuso, Nettetal, Steyler Verlag, 1994, p. 31-64; N. STANDAERT, « Jesuit corporate culture as shaped by the Chinese ", in The Jesuits. Cultures, sciences, and the arts, 1540-1773, éd. John W. O'MALLEY, Gauvin A. Balley, Steven J. Harris et T. Frank Kennedy, Toronto/Buffalo/Londres, University of Toronto Press, 1999.

24. "From Clavius to Pardies. The geometry transmitted to China by Jesuits (16071723) ", p. 175-199, in Western humanistic culture, op. cit. supra, p. 486.

25. Voir aussi « L'attitude des missionnaires jésuites face à l'astrologie et à la divination chinoises ", in op. cit. supra n. 21, éd. C. Jami et H. Delahaye, p. 87-108.

26. Voir aussi John WILLS, «Brief intersection. Changing contexts and prospects of the Chinese-Christian encounter from Matteo Ricci to Ferdinand Verbiest ", in op. cit. supra n. 1, p. 383-394. 
déplace donc de Beijing vers la campagne. Quel était, par exemple, l'état matériel d'une communauté jésuite en Chine au début du xvil ${ }^{e}$ siècle ${ }^{27}$ ? Et quelles étaient les dépenses du jésuite itinérant de campagne ${ }^{28}$. Ces nouvelles questions dépassent l'approche occidentale ou chinoise de la question, parce qu'elles s'appuient autant sur des sources en langues occidentales que chinoises et visent à une complémentarité de points de vue.

Nicolas StandaerT

(janvier 1999).

27. Voir Ad. Dudink, « The inventories of the Jesuit house at Nanjing, made up during the persecution of 1616-1617 ", p. 119-157, in Western humanistic culture, op. cit. supra, p. 486. 28. Voir N. Golvers, F. de Rougemont, S.J., missionary in Ch'ang-shu (Suchou). A study of the Account Book (1674-1676) and the Elogium, Leuven, Leuven University Press, 1999. 